Anuario Latinoamericano Ciencias Políticas

y Relaciones Internacionales

vol. 7, 2019

pp. 13-16

\section{Continuidad y cambio en el escenario regional de América Latina y el Caribe}

DOI: 10.17951/al.2019.7.13-16

\section{Continuity and Change in the Latin American and Caribbean Regional Scene}

Presentación

\author{
Katarzyna Krzywicka* \\ FACULTAD DE CIENCIAS POLÍTICAS Y PERIODISMO \\ UNIVERSIDAD MARIA CURIE-SKŁODOWSKA \\ LUBLIN, POLONIA \\ $\triangle$ katarzyna.krzywicka@umcs.edu.pl \\ https://orcid.org/0000-0001-5623-5384
}

La región de América Latina y el Caribe es un elemento importante del sistema internacional global que a fines de la segunda década del siglo XXI aparece como un área de cambios dinámicos. En la década de los años 90, en los países latinoamericanos empezaron procesos de transformaciones sistémicas políticas y económicas de carácter democrático y liberal, lo que influyó en la creciente importancia de la región en el sistema global de relaciones internacionales. Como resultado, América Latina y el Caribe se han convertido en una de las tres regiones del mundo -al lado de América del Norte y Europa- donde predominan los sistemas democráticos de gobernanza. Este es un fenómeno ya bien establecido, teniendo en cuenta que, entre los 33 Estados latinoamericanos, casi tres cuartos de ellos son democráticos. En los restantes países se nota la falta o cierto déficit en cuanto al respeto por los principios democráticos, así pues, en este aspecto se puede hablar de una erosión gradual de los sistemas democráticos y una disminución de la calidad de la democracia.

Un problema notable de las democracias latinoamericanas es la crisis de representación, la cual se expresa en la falta de confianza de los votantes en los

* Politóloga, Doctora en Humanidades por la Universidad Maria Curie-Skłodowska (UMCS) en Lublin, Polonia. Doctora habilitada en Ciencias Sociales por la Universidad de Wrocław, Polonia. Profesora, docente e investigadora del Departamento de Relaciones Internacionales de la Facultad de Ciencias Políticas y Periodismo de la UMCS en Lublin, Polonia.

Fundadora en 2011 y Directora Científica de la serie editorial Estudios Iberoamericanos de la UMCS. Fundadora en 2014 y Editora en Jefe de la revista Anuario Latinoamericano - Ciencias Politicas y Relaciones Internacionales publicada por la Facultad de Ciencias Políticas y Periodismo de la UMCS. 
Dossier América Latina: continuidad y cambio en el escenario regional partidos políticos. Esto se debe a que los partidos políticos tienen dificultades para adaptarse a los desafíos causados por los dinámicos cambios sociales, las crecientes expectativas de la clase media, la decepción por poca eficacia en la lucha contra la corrupción, la falta de la seguridad pública y de las soluciones a los problemas de desigualdad social en los países de la región.

El ciclo electoral 2017-2019 trajo un cambio en las preferencias electorales y, en algunos Estados de América Latina, los líderes políticos populistas y antisistema obtuvieron apoyo social. Este fenómeno viene acompañado por un aumento de la polarización social y una clara caída de apoyo a la democracia que, según las encuestas de opinión pública, ha alcanzado el 12\% en la última década. Por otro lado, los países de América Latina mantienen un alto nivel de participación electoral en comparación con otras regiones del mundo, lo que se debe indudablemente también al hecho de que en 14 Estados latinoamericanos la participación en las elecciones es obligatoria.

En la última década del siglo XX, los países de América Latina y el Caribe experimentaron cambios económicos, pasando del neoliberalismo a la implementación de proyectos posliberales en la primera década del siglo XXI. Han surgido nuevas formas de integración regional sin la participación de los Estados Unidos de América. Por otro lado, algunos países de América Central y la región andina continúan implementando proyectos neoliberales en cooperación con los Estados Unidos. El papel tradicional de los países latinoamericanos como base global de recursos naturales no ha cambiado. Las economías de los países de la región siguen dependiendo de la exportación de materias primas y son sensibles a los cambios en sus precios en los mercados mundiales. Teniendo en cuenta los fenómenos políticos y económicos mencionados, se puede notar una clara correlación de dos tendencias principales en la región, a saber, la decepción de los ciudadanos tanto por el neoliberalismo como por la democracia.

Los autores de los artículos del Dossier analizan los procesos de cambio que tienen lugar en la región de América Latina, adoptando dos perspectivas de investigación. En primer lugar, se refieren a las principales tendencias políticas, económicas, sociales y culturales que dominan en la región y, en segundo lugar, llevan a cabo el análisis del Estado desde el nivel sistémico. Reflexionan sobre los condicionamientos, desafíos y amenazas a la estabilidad económica y política de los países de la región; formas de cooperación política, económica y cultural regional e interregional; las particularidades del liderazgo político; la especificidad de la transformación de los sistemas políticos; la naturaleza y características de las amenazas a la seguridad. Los autores tratan los problemas políticos y socioeconómicos en los países latinoamericanos, analizándolos como elementos de la seguridad ampliamente entendida.

En el artículo La dimensión político-institucional de los procesos de integración de América Latina (2000-2016), Alberto Rocha Valencia analiza las cuestiones de la gobernabilidad y gobernanza en los planos regional y subregional del proceso de integración de la región, con la finalidad de explorar la relación 
entre la gobernabilidad y gobernanza de los esquemas político-institucionales de los procesos de integración subregionales y el esquema de gobernabilidad y gobernanza del proceso de integración regional.

En el siguiente artículo, Geneviève Marchini caracteriza el funcionamiento de la Alianza del Pacífico a ocho años de su creación. La autora dedica su análisis a las expectativas que generó la Alianza en materia de integración intrarregional y extrarregional y a las condiciones necesarias para su éxito. Además, discute las perspectivas de este mecanismo a la luz de acontecimientos y cambios recientes en la economía global.

A su vez, María de Monserrat Llairó, continuando con el tema de la integración regional, analiza los nuevos desafíos y ejes de poder de la integración latinoamericana, concentrándose en la dualidad Mercosur - Alianza del Pacífico durante el periodo 2010-2017. La autora intenta explicar los procesos de la integración regional en un entorno cambiante, en vista de la globalización y de los cambios políticos e institucionales en la región de América Latina.

Katarzyna Marzęda-Młynarska, en el artículo titulado La gobernanza de la seguridad alimentaria en América Latina: desde la cooperación Norte-Sur hacia la cooperación Sur-Sur, analiza el modelo latinoamericano de gobernanza de la seguridad alimentaria con especial énfasis en el papel de las organizaciones regionales, demostrando que el modelo regional de gobernanza de la seguridad alimentaria se basa en el mecanismo de cooperación Sur-Sur, el cual promueve la cooperación y las acciones conjuntas de los países en desarrollo para resolver el problema del hambre y desnutrición. La investigación abarca el periodo relacionado con la implementación de la Agenda 2030, en la cual la cooperación Sur-Sur ha sido reconocida como un instrumento importante para alcanzar los Objetivos de Desarrollo Sostenible.

El artículo de Gerardo Reyes Guzmán, titulado Relaciones comerciales México-Estados Unidos de América: 2016-2018, es un análisis de las principales características del comercio entre ambos países vecinos durante la administración de Donald Trump. Partiendo del enrarecimiento de la relación comercial reflejado en la volatilidad del tipo de cambio y los compromisos acordados en el Tratado México-Estados Unidos-Canadá, el autor explica los factores que determinan el nuevo proteccionismo-nacionalismo de los Estados Unidos.

La política exterior de los Estados Unidos de América es también un tema de reflexión de Ieva Giedraityte. La autora, en su artículo titulado Empire, Leadership or Hegemony: US Strategies towards the Northern Triangle Countries in the $21^{\text {st }}$ Century, sistematiza y analiza las estrategias de los EE. UU. hacia Honduras, Guatemala y El Salvador, los países del Triángulo Norte, siguiendo la categorización de Destradi. El análisis lleva a una conclusión que la estrategia de los EE. UU. hacia la región puede definirse como hegemónica, sin embargo, progresivamente se ha cambiado de dura a intermedia, a medida que los EE. UU. se hicieron más perceptivos a las necesidades de sus socios regionales.

En el siguiente artículo, titulado La reformulación de la política exterior argentina en el gobierno de Macri: el difícil equilibrio entre los cambios globales 
Dossier América Latina: continuidad y cambio en el escenario regional y las crisis domésticas (2015-2018), su autora Priscila Palacio analiza la política exterior del gobierno de Mauricio Macri, destacando las restricciones que impuso la economía doméstica. Se indaga sobre las causas que han generado el resurgimiento del proteccionismo o nacionalismo económico, en detrimento de la globalización y neoliberalismo institucional. La autora analiza los aspectos más relevantes de la política exterior de Argentina entre 2015 y 2018, el período en el cual se definieron los ejes fundamentales de la gestión política.

Los autores Maria do Socorro Sousa Braga y Gabriel Avila Casalecchi, en su artículo titulado Legitimidad y compromiso democrático. Impases contemporáneos en América Latina, analizan algunas actitudes políticas de los ciudadanos y su relación con los cambios en el régimen político para identificar cuál es el nivel de la reserva de legitimidad política y de compromiso con la democracia en la región. Los autores concluyen que la combinación de una baja legitimidad y compromiso democrático con la percepción negativa de la efectividad de las instituciones y del gobierno sirve como una alerta para la democracia en América Latina.

El objetivo del artículo escrito por Marta Kania, titulado Qhapaq Nan: Indigenous Peoples' Heritage as an Instrument of Inter-American Integration Poli$c y$, es de presentar las relaciones entre los países de América del Sur en cuanto a la política de la protección y salvaguarda de uno de los elementos más excepcionales del patrimonio cultural de los pueblos indígenas de la región, representado por el Sistema Vial Andino - Qhapaq Ñan. La autora comparte algunas reflexiones sobre el estado actual de la política cultural y el proceso de implementación de los derechos de los pueblos indígenas en relación con el derecho a la participación y la gestión de su patrimonio cultural.

Marta Zuzanna Osuchowska, en el artículo titulado América Latina como la región de transformaciones religiosas en el siglo XXI, analiza los cambios que se están produciendo en el ámbito religioso en la región, con especial énfasis en los aspectos legales de este fenómeno. Las constituciones de los Estados latinoamericanos consideran la libertad religiosa en diversos grados. Las dificultades suelen residir en actos y disposiciones administrativas que protegen este derecho y regulan su expresión en el espacio público. La autora indica principales tendencias regionales en relación con los cambios que tienen lugar en el espacio religioso en los países de América Latina y el Caribe.

La lectura de los artículos del Dossier lleva a la conclusión que América Latina no es una región homogénea a pesar de los fuertes lazos históricos y culturales, como el idioma y la religión, que comparten los países latinoamericanos. La diversidad se expresa en diferentes prioridades políticas y económicas, los cuales se cambian dinámicamente, junto con ciclos electorales sucesivos. Tal situación implica una disminución de la capacidad de cooperar, lo cual se evidencia en baja eficiencia de los proyectos de integración emprendidos en América Latina y el Caribe. Además, afecta la posibilidad de cooperación en otros campos relevantes para la seguridad de los Estados de la región. 